Dokuz Eylül Üniversitesi-Mühendislik Fakültesi Fen ve Mühendislik Dergisi

Cilt 19 Sayı 55 Ocak 2017
Dokuz Eylul University-Faculty of Engineering Journal of Science and Engineering Volume 19 Issue 55 January 2017

DOI: $10.21205 /$ deufmd. 2017195520

\title{
Characteristic Properties of the New Subclasses of Analytic Functions
}

\author{
Nizami MUSTAFA \\ Department of Mathematics, Faculty of Science and Letters, Kafkas University, 36100, Kars \\ (Alınış / Received: 12.10.2016, Kabul / Accepted: 24.11.2016, \\ Online Yayınlanma / Published Online: 09.01.2017) \\ Keywords \\ Analytic function, \\ Starlike function, \\ Convex function \\ Abstract: In this study, we introduce and investigate two new \\ subclasses of analytic functions in the open unit disk. The object of \\ the present paper is to derive characteristic properties of the \\ functions belonging to these classes. Further, several coefficient \\ inequalities for the functions belonging to these classes are also \\ given.
}

\section{Analitik Fonksiyonların Yeni Alt Sınıflarının}

\section{Karakteristik Özellikleri}

\begin{abstract}
Anahtar kelimeler Özet: Bu çalışmada biz açık birim diskte analitik fonksiyonların iki Analitik fonksiyon, Yıldızıl fonksiyon, Konveks fonksiyon yeni altsınıfını tanımladık ve araștırdık. Mevcut çalıșmanın amacı bu sınıflara ait fonksiyonların karakteristik özelliklerini elde etmektir. Dahası, bu sınıflara ait olan fonksiyonlar için çeşitli katsayı eşitsizlikleri de verilmiştir.
\end{abstract}

Corresponding author: nizamimustafa@gmail.com 


\section{Introduction and Preliminaries}

Let $A$ be the class of analytic functions $f(z)$ in the open unit disk $U=\{z \in \square:|z|<1\}$, normalized by $f(0)=0=f^{\prime}(0)-1$ of the form

$$
f(z)=z+a_{2} z^{2}+\cdots+a_{n} z^{n}+\cdots
$$$$
=z+\sum_{n=2}^{\infty} a_{n} z^{n}, a_{n} \in \square \text {. }
$$

Further, by $S$ we will denote the family of all functions in $A$ which are univalent in $U$.

Let $T$ denote the subclass of all functions $f(z)$, with non-positive coefficients, in $A$ of the form

$$
\begin{aligned}
& f(z)=z-a_{2} z^{2}-\cdots-a_{n} z^{n}-\cdots \\
& =z-\sum_{n=2}^{\infty} a_{n} z^{n}, a_{n} \geq 0
\end{aligned}
$$

Many researchers have introduced and investigated several subclasses of analytic function class $A$ (see, for example [1-3]). Various subclasses of $A$ were introduced and some geometric properties of these subclasses were investigated in several studies (see [4-7]).

Recently, Prajapat [8] introduced the subclasses

$R_{a}^{\lambda}(k, \alpha), V_{a}^{\lambda}(k, \alpha), T_{a}^{\lambda}(k, \beta, \alpha)$

and $\bar{T}_{a}^{\lambda}(k, \beta, \alpha)$ of $A$ and several inclusion relationships were established for these subclasses. Also, very soon Prajapat [9] introduced an interesting subclass $\chi_{t}(\gamma)$ of analytic and close-to- convex functions in the open unit disk $U$. In [9], Prajapat derived several properties including coefficients estimates, distortion theorems, covering theorems and radius of convexity for the functions belonging to the class $\chi_{t}(\gamma)$.

Soon after that, Mustafa [10] introduced and investigated the subclass $K(\alpha, \beta), \alpha, \beta \in[0,1)$, which is the generalization of the close-to-convex functions class, named close-to-convex with respect to a starlike function $g(z)$ of order $\alpha(\alpha \in[0,1))$ and type $\beta(\beta \in[0,1))$ of analytic functions in the open unit disk $U$. In [10], Mustafa found sufficient conditions for the parameters of the normalized Wright functions to be in the class $K(\alpha, \beta)$.

Very recently, Panigrahi and Murugusundaramoorthy [11] have introduced a new subclass of the univalent functions class $S$, denoted by $M_{\lambda, \delta}^{k, t}(\alpha)$ and they have found sharp estimates for the difference of the coefficients of the functions belonging to this class.

As it can be seen from the above mentioned studies, some of the important and well-investigated subclasses of $S$ are the classes $S^{*}(\alpha)$ and $C(\alpha)$ defined as follows.

Definition 1.1. (see also [12-14]) The class of starlike functions $S^{*}(\alpha)$ of order $\alpha(\alpha \in[0,1))$ and the class of convex functions $C(\alpha)$ of order 
$\alpha(\alpha \in[0,1))$ are defined, respectively,

by

$S^{*}(\alpha)=$

$=\left\{\begin{array}{l}f \in A: \operatorname{Re}\left(\frac{z f^{\prime}(z)}{f(z)}\right)>\alpha, \\ z \in U\end{array}\right\}$,

$\alpha \in[0,1)$

and

$C(\alpha)=$

$=\left\{\begin{array}{l}f \in A: \operatorname{Re}\left(1+\frac{z f^{\prime \prime}(z)}{f^{\prime}(z)}\right)>\alpha, \\ z \in U\end{array}\right\}$,

$\alpha \in[0,1)$.

We will denote

$T S^{*}(\alpha)=S^{*}(\alpha) \cap T$

and

$T C(\alpha)=C(\alpha) \cap T$.

Interesting generalization of the functions classes $S^{*}(\alpha)$ and $C(\alpha)$ are denoted, respectively, by $S^{*}(\alpha, \beta)$ and $C(\alpha, \beta)$, and defined by

$S^{*}(\alpha, \beta)=$

$\left\{\begin{array}{l}f \in A: \\ \operatorname{Re}\left(\frac{z f^{\prime}(z)}{\beta z f^{\prime}(z)+(1-\beta) f(z)}\right)>\alpha, \\ z \in U\end{array}\right\}$,

$\alpha, \beta \in[0,1)$ and

$C(\alpha, \beta)=$

$=\left\{\begin{array}{l}f \in A: \\ \operatorname{Re}\left(\frac{f^{\prime}(z)+z f^{\prime \prime}(z)}{f^{\prime}(z)+\beta z f^{\prime \prime}(z)}\right)>\alpha, \\ z \in U\end{array}\right\}$,

$\alpha, \beta \in[0,1)$.

Moreover, we will denote

$T S^{*}(\alpha, \beta)=S^{*}(\alpha, \beta) \cap T$

and

$T C(\alpha, \beta)=C(\alpha, \beta) \cap T$.

The classes $T S^{*}(\alpha, \beta)$ and $T C(\alpha, \beta)$ were extensively studied by Altıntaş and Owa [15] and certain conditions for hypergeometric functions and generalized Bessel functions for these classes were studied by Moustafa [16] and by Porwal and Dixit [17].

Inspired by the above mentioned studies, we define a unification of the functions classes $S^{*}(\alpha, \beta)$ and $C(\alpha, \beta)$ as follows.

Definition 1.2. A function $f \in A$ given by (1) is said to be in the class $S^{*} C(\alpha, \beta ; \gamma)$ $(\alpha, \beta \in[0,1), \gamma \in[0,1])$ if the following condition is satisfied 
$\operatorname{Re}\left\{\begin{array}{l}\frac{z f^{\prime}(z)+\gamma z^{2} f^{\prime \prime}(z)}{\gamma z\left(f^{\prime}(z)+\beta z f^{\prime \prime}(z)\right)+} \\ \frac{(1-\gamma)\left(\beta z f^{\prime}(z)+(1-\beta) f(z)\right)}{\alpha}\end{array}\right\}$

$>\alpha, z \in U$.

Further, we will use

$$
T S^{*} C(\alpha, \beta ; \gamma)=S^{*} C(\alpha, \beta ; \gamma) \cap T \text {. }
$$

In special case, we have

$$
\begin{aligned}
& S^{*} C(\alpha, \beta ; 0)=S^{*}(\alpha, \beta) ; \\
& S^{*} C(\alpha, \beta ; 1)=C(\alpha, \beta) ; \\
& S^{*} C(\alpha, 0 ; 0)=S^{*}(\alpha) ; \\
& S^{*} C(\alpha, 0 ; 1)=C(\alpha) ; \\
& T S^{*} C(\alpha, \beta ; 0)=T S^{*}(\alpha, \beta) ; \\
& T S^{*} C(\alpha, \beta ; 1)=T C(\alpha, \beta) ; \\
& T S^{*} C(\alpha, 0 ; 0)=T S^{*}(\alpha) ; \\
& T S^{*} C(\alpha, 0 ; 1)=T C(\alpha) .
\end{aligned}
$$

Suitably specializing the parameters we note that

1) $S^{*} C(\alpha, 0 ; 0)=S^{*}(\alpha)[18]$;

2) $S^{*} C(\alpha, 0 ; 1)=C(\alpha)[18]$;

3) $T S^{*} C(\alpha, \beta ; 0)=T S^{*}(\alpha, \beta)[19$ 22];

4) $T S^{*} C(\alpha, 0 ; 0)=T S^{*}(\alpha)[18]$;

5) $T S^{*} C(\alpha, \beta ; 1)=T C(\alpha, \beta)$ [15];

6) $T S^{*} C(\alpha, 0 ; 1)=T C(\alpha)[18]$.

The object of the present paper is to examine characteristic properties of the classes
$S^{*} C(\alpha, \beta ; \gamma)$ and $T S^{*} C(\alpha, \beta ; \gamma)$,

$\alpha, \beta \in[0,1), \gamma \in[0,1]$. In this paper, coefficient bounds for the functions belonging in these classes are also determined.

\section{Coefficient Bounds for the Classes $S^{*} C(\alpha, \beta ; \gamma)$ and $T S^{*} C(\alpha, \beta ; \gamma)$}

In this section, we will examine some characteristic properties of the classes

$$
\begin{aligned}
& S^{*} C(\alpha, \beta ; \gamma) \text { and } T S^{*} C(\alpha, \beta ; \gamma) \\
& (\alpha, \beta \in[0,1), \gamma \in[0,1])
\end{aligned}
$$

of analytic functions in the open unit disk $U$. Here, coefficient bounds for the functions belonging to these classes are also given.

A sufficient condition for the functions in the class

$S^{*} C(\alpha, \beta ; \gamma), \alpha, \beta \in[0,1), \gamma \in[0,1]$

is given by the following theorem.

Theorem 2.1. Let $f \in A$. Then, the function $f(z)$ belongs to the class

$$
S^{*} C(\alpha, \beta ; \gamma)(\alpha, \beta \in[0,1), \gamma \in[0,1])
$$

if the following condition is satisfied

$$
\sum_{n=2}^{\infty}\left\{\begin{array}{l}
(1+(n-1) \gamma) \\
\times(n-\alpha-(n-1) \alpha \beta)
\end{array}\right\}\left|a_{n}\right| \leq 1-\alpha .
$$

The result is sharp for the functions 
$f_{n}(z)=z+$

$\frac{(1-\alpha) z^{n}}{(1+(n-1) \gamma)(n-\alpha-(n-1) \alpha \beta)}$,

$z \in U, n=2,3, \ldots$.

Proof. Let

$f \in S^{*} C(\alpha, \beta ; \gamma), \alpha, \beta \in[0,1)$,

$\gamma \in[0,1]$. Then, according to Definition

1.2 , we have

$\operatorname{Re}\left\{\begin{array}{l}\frac{z f^{\prime}(z)+\gamma z^{2} f^{\prime \prime}(z)}{\gamma z\left(f^{\prime}(z)+\beta z f^{\prime \prime}(z)\right)+} \\ \frac{(1-\gamma)\left(\beta z f^{\prime}(z)+(1-\beta) f(z)\right)}{}\end{array}\right\}$

$>\alpha, z \in U$.

Also, we can easily show that the condition (5) holds true if

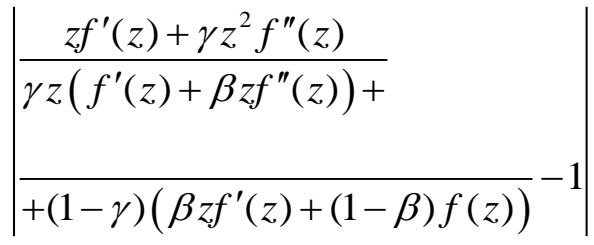

$$
\begin{aligned}
& \leq 1-\alpha \text {. }
\end{aligned}
$$

(6)

Therefore, for the complete the proof of the theorem suffices to show that the condition (6) is satisfied.

By simple computation, we obtain $\left|\begin{array}{l}\frac{z f^{\prime}(z)+\gamma z^{2} f^{\prime \prime}(z)}{\gamma z\left(f^{\prime}(z)+\beta z f^{\prime \prime}(z)\right)+} \\ +(1-\gamma)\left(\beta z f^{\prime}(z)+(1-\beta) f(z)\right) \\ -1\end{array}\right|=$

$$
\begin{aligned}
&=\left|\frac{\sum_{n=2}^{\infty}\left[\begin{array}{l}
(1+(n-1) \gamma) \\
\times(n-1)(1-\beta)
\end{array}\right] a_{n} z^{n}}{z+}\right| \\
& \leq\left.\frac{\sum_{n=2}^{\infty}\left[\begin{array}{l}
(1+(n-1) \gamma) \\
\times(1+(n-1) \beta)
\end{array}\right] a_{n} z^{n}}{\times} \mid \begin{array}{l}
(1+(n-1) \gamma) \\
\times(n-1)(1-\beta)
\end{array}\right]\left|a_{n}\right| \\
& 1-\sum_{n=2}^{\infty}\left[\begin{array}{l}
(1+(n-1) \gamma) \\
\times(1+(n-1) \beta)
\end{array}\right]\left|a_{n}\right|
\end{aligned} .
$$

Last expression of the above inequality is bounded by $1-\alpha$ if

$$
\begin{aligned}
& \sum_{n=2}^{\infty}(1+(n-1) \gamma)(n-1)(1-\beta)\left|a_{n}\right| \\
& \leq(1-\alpha) \\
& \times\left\{1-\sum_{n=2}^{\infty}\left[\begin{array}{l}
(1+(n-1) \gamma) \\
\times(1+(n-1) \beta)
\end{array}\right]\left|a_{n}\right|\right\},
\end{aligned}
$$

which is equivalent to (3).

We can easily see that the result of the theorem is sharp for the functions given by (4).

Thus, the proof of Theorem 2.1 is completed. 
By setting $\gamma=0$ and $\gamma=1$ in Theorem 2.1 , we can readily deduce the following results.

Corollary 2.1. The function $f(z)$ defined by (1) belongs to the class $S^{*}(\alpha, \beta)(\alpha, \beta \in[0,1))$ if the following condition is satisfied

$\sum_{n=2}^{\infty}(n-\alpha-(n-1) \alpha \beta)\left|a_{n}\right| \leq 1-\alpha$.

The result is sharp for the functions

$$
\begin{aligned}
& f_{n}(z)=z+\frac{1-\alpha}{n-\alpha-(n-1) \alpha \beta} z^{n}, \\
& z \in U, n=2,3, \ldots
\end{aligned}
$$

Corollary 2.2. The function $f(z)$ defined by (1) belongs to the class $C(\alpha, \beta)(\alpha, \beta \in[0,1))$ if the following condition is satisfied

$$
\sum_{n=2}^{\infty} n(n-\alpha-(n-1) \alpha \beta)\left|a_{n}\right|
$$

$\leq 1-\alpha$.

The result is sharp for the functions

$$
\begin{aligned}
& f_{n}(z)=z+ \\
& +\frac{1-\alpha}{n(n-\alpha-(n-1) \alpha \beta)} z^{n}, \\
& z \in U, n=2,3, \ldots .
\end{aligned}
$$

By taking $\beta=0$ in Corollary 2.1 and 2.2 , respectively, we have the following results.

Corollary 2.3. (see [18, p. 110, Theorem 1]) The function $f(z)$ defined by (1) belongs to the class
$S^{*}(\alpha)(\alpha \in[0,1))$ if the following condition is satisfied

$$
\sum_{n=2}^{\infty}(n-\alpha)\left|a_{n}\right| \leq 1-\alpha .
$$

The result is sharp for the functions

$$
\begin{aligned}
& f_{n}(z)=z+\frac{1-\alpha}{n-\alpha} z^{n}, z \in U, \\
& n=2,3, \ldots
\end{aligned}
$$

Corollary 2.4. (see [18, p. 110, Corollary of Theorem 1]) The function $f(z)$ defined by (1) belongs to the class $C(\alpha)(\alpha \in[0,1))$ if the following condition is satisfied

$$
\sum_{n=2}^{\infty} n(n-\alpha)\left|a_{n}\right| \leq 1-\alpha .
$$

The result is sharp for the functions

$$
\begin{aligned}
& f_{n}(z)=z+\frac{1-\alpha}{n(n-\alpha)} z^{n}, z \in U, \\
& n=2,3, \ldots
\end{aligned}
$$

Remark 2.1. Numerous consequences of the properties given by Corollary 2.3 and 2.4 can be obtained for each of the classes studied by earlier researchers, by specializing the various parameters involved. Many of these consequences were proved by earlier researches on the subject (cf., e.g., [18]).

For the function in the class $T S^{*} C(\alpha, \beta ; \gamma)$, the converse of Theorem 2.1 is also true.

Theorem 2.2. Let $f \in T$. Then, the function $f(z)$ belongs to the class 
$T S^{*} C(\alpha, \beta ; \gamma)$

$(\alpha, \beta \in[0,1), \gamma \in[0,1])$

if and only if

$\sum_{n=2}^{\infty}\left[\begin{array}{l}(1+(n-1) \gamma) \\ \times(n-\alpha-(n-1) \alpha \beta)\end{array}\right] a_{n}$

$\leq 1-\alpha$.

The result is sharp for the functions

$$
\begin{aligned}
& f_{n}(z)=z- \\
& \frac{(1-\alpha) z^{n}}{(1+(n-1) \gamma)(n-\alpha-(n-1) \alpha \beta)}, \\
& z \in U, n=2,3, \ldots
\end{aligned}
$$

Proof. The proof of the sufficiency of the theorem can be proved similarly to the proof of Theorem 2.1.

We will prove only the necessity of the theorem. Assume that

$f \in T S^{*} C(\alpha, \beta ; \gamma), \alpha, \beta \in[0,1)$,

$\gamma \in[0,1]$. That is,

$\operatorname{Re}\left\{\begin{array}{l}\frac{z f^{\prime}(z)+\gamma z^{2} f^{\prime \prime}(z)}{\gamma z\left(f^{\prime}(z)+\beta z f^{\prime \prime}(z)\right)+} \\ +(1-\gamma)\left(\begin{array}{l}\beta z f^{\prime}(z)+ \\ +(1-\beta) f(z)\end{array}\right)\end{array}\right\}>\alpha$,

$z \in U$.

By simple computation, we obtain

$$
\begin{aligned}
& \operatorname{Re}\left\{\begin{array}{l}
\frac{z f^{\prime}(z)+\gamma z^{2} f^{\prime \prime}(z)}{\gamma z\left(f^{\prime}(z)+\beta z f^{\prime \prime}(z)\right)+} \\
+(1-\gamma)\left(\begin{array}{l}
\beta z f^{\prime}(z)+ \\
+(1-\beta) f(z)
\end{array}\right)
\end{array}\right\} \\
& =\operatorname{Re}\left\{\begin{array}{l}
\frac{z-\sum_{n=2}^{\infty} n(1+(n-1) \gamma) a_{n} z^{n}}{z-} \\
-\sum_{n=2}^{\infty}\left[\begin{array}{l}
(1+(n-1) \gamma) \\
\times(1+(n-1) \beta)
\end{array}\right] a_{n} z^{n}
\end{array}\right\}
\end{aligned}
$$

$>\alpha$.

The last expression in the brackets of the above inequality is real if we choose $z$ as a real. Hence, from the previous inequality letting $z \rightarrow 1$ through real values, we obtain

$$
\begin{aligned}
& 1-\sum_{n=2}^{\infty} n[1+\gamma(n-1)] a_{n} \geq \\
& \geq \alpha\left\{1-\sum_{n=2}^{\infty}\left[\begin{array}{c}
(1+(n-1) \gamma) \\
\times(1+\beta(n-1))
\end{array}\right] a_{n}\right\} .
\end{aligned}
$$

It follows that

$$
\sum_{n=2}^{\infty}\left[\begin{array}{l}
(1+(n-1) \gamma) \\
\times(n-\alpha-(n-1) \alpha \beta)
\end{array}\right] a_{n} \leq 1-\alpha,
$$

which is the same as the condition (7).

Moreover, it is clear that the equality in (7) is satisfied by the functions given by (8).

Thus, the proof of Theorem 2.2 is completed. 
By taking $\gamma=0$ and $\gamma=1$ in Theorem 2.2 , we can readily deduce the following results.

Corollary 2.5. The function $f(z)$ defined by (2) belongs to the class $T S^{*}(\alpha, \beta)(\alpha, \beta \in[0,1))$ if and only if $\sum_{n=2}^{\infty}(n-\alpha-(n-1) \alpha \beta) a_{n} \leq 1-\alpha$

The result is sharp for the functions

$$
\begin{aligned}
& f_{n}(z)=z-\frac{1-\alpha}{n-\alpha-(n-1) \alpha \beta} z^{n}, \\
& z \in U, n=2,3, \ldots .
\end{aligned}
$$

Corollary 2.6. The function $f(z)$ defined by (2) belongs to the class $T C(\alpha, \beta)(\alpha, \beta \in[0,1))$ if and only if $\sum_{n=2}^{\infty} n(n-\alpha-(n-1) \alpha \beta) a_{n} \leq 1-\alpha$.

The result is sharp for the functions

$$
\begin{aligned}
& f_{n}(z)=z- \\
& -\frac{1-\alpha}{n(n-\alpha-(n-1) \alpha \beta)} z^{n}, \\
& z \in U, n=2,3, \ldots .
\end{aligned}
$$

Remark 2.2. The results obtained by Corollary 2.5 and Corollary 2.6 would reduce to known results in [15].

By taking $\beta=0$ in Corollary 2.5 and 2.6 , respectively, we have the following results.

Corollary 2.7. (see [18, p. 110, Theorem 2]) The function $f(z)$ defined by (2) belongs to the class $T S^{*}(\alpha)(\alpha \in[0,1))$ if and only if

$$
\sum_{n=2}^{\infty}(n-\alpha) a_{n} \leq 1-\alpha
$$

The result is sharp for the functions

$$
\begin{aligned}
& f_{n}(z)=z-\frac{1-\alpha}{n-\alpha} z^{n}, \\
& z \in U, n=2,3, \ldots .
\end{aligned}
$$

Corollary 2.8. (see [18, p. 111, Corollary 2]) The function $f(z)$ defined by (2) belongs to the class $\operatorname{TC}(\alpha)(\alpha \in[0,1))$ if and only if

$$
\sum_{n=2}^{\infty} n(n-\alpha) a_{n} \leq 1-\alpha .
$$

The result is sharp for the functions

$$
\begin{aligned}
& f_{n}(z)=z-\frac{1-\alpha}{n(n-\alpha)} z^{n}, \\
& z \in U, n=2,3, \ldots
\end{aligned}
$$

From Theorem 2.2, we have the following result.

\section{Corollary 2.9. If}

$f \in T S^{*} C(\alpha, \beta ; \gamma)$, then

$$
\left|a_{n}\right| \leq \frac{1-\alpha}{\left[\begin{array}{l}
(1+(n-1) \gamma) \\
\times(n-\alpha-(n-1) \alpha \beta)
\end{array}\right]},
$$

$n=2,3, \ldots$.

Remark 2.3. Numerous consequences of Corollary 2.9 can be deduced by specializing the various parameters involved. Many of these consequences 
were proved by earlier researchers on the subject (cf., e.g., $[18,19])$.

On the coefficient bounds of the functions belonging in the class $T S^{*} C(\alpha, \beta ; \gamma)$, we give the following theorem.

Theorem 2.3. Let the function $f(z)$ defined by (2) belongs to the class

$T S^{*} C(\alpha, \beta ; \gamma)$

$(\alpha, \beta \in[0,1), \gamma \in[0,1])$.

Then,

$$
\sum_{n=2}^{\infty}\left|a_{n}\right| \leq \frac{1-\alpha}{(1+\gamma)(2-(1+\beta) \alpha)}
$$

and

$$
\sum_{n=2}^{\infty} n\left|a_{n}\right| \leq \frac{2(1-\alpha)}{(1+\gamma)(2-(1+\beta) \alpha)}
$$

Proof. Using Theorem 2.2, we write

$$
\begin{aligned}
& (1+\gamma)(2-(1+\beta) \alpha) \sum_{n=2}^{\infty}\left|a_{n}\right| \\
& \leq \sum_{n=2}^{\infty}\left[\begin{array}{l}
(1+(n-1) \gamma) \\
\times(n-\alpha-(n-1) \alpha \beta)
\end{array}\right]\left|a_{n}\right| \\
& \leq 1-\alpha .
\end{aligned}
$$

That is,

$$
\sum_{n=2}^{\infty}\left|a_{n}\right| \leq \frac{1-\alpha}{(1+\gamma)(2-(1+\beta) \alpha)} .
$$

Thus, inequality (9) is provided.

Similarly, we obtain

$$
\begin{aligned}
& (2-(1+\beta) \alpha) \sum_{n=2}^{\infty}(1-\gamma+n \gamma)\left|a_{n}\right| \\
& \leq \sum_{n=2}^{\infty}\left[\begin{array}{l}
(1+(n-1) \gamma) \\
\times(n-\alpha-(n-1) \alpha \beta)
\end{array}\right]\left|a_{n}\right| \\
& \leq 1-\alpha,
\end{aligned}
$$

which is equivalent to

$$
\begin{aligned}
& (2-(1+\beta) \alpha) \gamma \sum_{n=2}^{\infty} n\left|a_{n}\right| \\
& \leq 1-\alpha-(1-\gamma)(2-(1+\beta) \alpha) \sum_{n=2}^{\infty}\left|a_{n}\right| .
\end{aligned}
$$

Using (9) in the last inequality, we arrive at the following

$$
\begin{aligned}
& (2-(1+\beta) \alpha) \gamma \sum_{n=2}^{\infty} n\left|a_{n}\right| \\
& \leq 1-\alpha-(1-\gamma)(2-(1+\beta) \alpha) \\
& \times \frac{1-\alpha}{(1+\gamma)(2-(1+\beta) \alpha)}=\frac{2 \gamma(1-\alpha)}{1+\gamma} .
\end{aligned}
$$

This immediately yields the second assertion (10) of Theorem 2.3.

By setting $\gamma=0$ and $\gamma=1$ in Theorem 2.3 , we arrive at the following results, respectively.

Corollary 2.10. Let the function $f(z)$ defined by (2) belongs to the class

$\operatorname{TS}^{*}(\alpha, \beta)(\alpha, \beta \in[0,1))$.

Then,

$\sum_{n=2}^{\infty}\left|a_{n}\right| \leq \frac{1-\alpha}{2-(1+\beta) \alpha}$ and $\sum_{n=2}^{\infty} n\left|a_{n}\right| \leq \frac{2(1-\alpha)}{2-(1+\beta) \alpha}$. 
Corollary 2.11. Let the function $f(z)$ defined by (2) belongs to the class

$\operatorname{TC}(\alpha, \beta)(\alpha, \beta \in[0,1))$.

Then,

$$
\begin{aligned}
& \sum_{n=2}^{\infty}\left|a_{n}\right| \leq \frac{1-\alpha}{2(2-(1+\beta) \alpha)} \text { and } \\
& \sum_{n=2}^{\infty} n\left|a_{n}\right| \leq \frac{1-\alpha}{2-(1+\beta) \alpha} .
\end{aligned}
$$

Remark 2.4. Numerous consequences of the coefficient inequalities (given by Corollary 2.10 and Corollary 2.11) can indeed be deduced by specializing the various parameters involved. For example, by setting $\beta=0$, we obtain the results for the classes $T S^{*}(\alpha)$ and $T C(\alpha)(\alpha \in[0,1))$, respectively.

Moreover, by setting $\alpha=\beta=0$ in Corollary 2.10 and 2.11 , we obtain interesting results for the classes $T S^{*}$ and $T C$, respectively.

Corollary 2.12. Let the function $f(z)$ defined by (2) belongs to the class $T S^{*}$. Then,

$\sum_{n=2}^{\infty}\left|a_{n}\right| \leq \frac{1}{2}$ and $\sum_{n=2}^{\infty} n\left|a_{n}\right| \leq 1$

Corollary 2.13. Let the function $f(z)$ defined by (2) belongs to the class $T C$. Then,

$$
\sum_{n=2}^{\infty}\left|a_{n}\right| \leq \frac{1}{4} \text { and } \sum_{n=2}^{\infty} n\left|a_{n}\right| \leq \frac{1}{2}
$$

\section{Concludıng Remarks}

In this paper, two new subclasses $S^{*} C(\alpha, \beta ; \gamma)$ and $T S^{*} C(\alpha, \beta ; \gamma)$, $\alpha, \beta \in[0,1), \gamma \in[0,1]$ of analytic functions in the open unit disk are introduced and investigated. In the present paper, the characteristic properties of the functions belonging to these classes are derived. Further, several coefficient inequalities for functions belonging to these classes are obtained.

\section{Acknowledgment}

The author is grateful to the anonymous referees for the valuable comments and suggestions.

\section{References}

[1] Frasin, B. A. 2012. New subclasses of analytic functions: Journal of Inequalities and Applications, Volume 24, pp. 1-10.

[2] Cho, N. E. and Kim, J. A. 2006. Inclusion Properties of Certain Subclasses of Analytic Functions Defined by a Multiplier Transformation: Computers and Mathematics with Applications, Volume 52, pp. 323-330.

[3] Darus, M. and Faisal, I. 2012. Some subclasses of analytic functions of complex order defined by new differential operator: Tamkang Journal of Mathematics, Volume 43, pp. 223-242.

[4] Gao, C. Y., Zhou, S. Q. 2005. On a class of analytic functions related to the class to the starlike functions: Kyungpook Mathematical Journal, Volume 45, pp. 123-130.

[5] Kowalczyk, J., Les-Bomba, E. 2010. On a subclass of close-to-convex functions: Applied Mathematics Letters, Volume 23, pp. 1147-1151. 
[6] Xu, Q. H., Srivastava, H. M., Li, Z. 2011. A certain subclass of analytic and close-to-convex functions: Applied Mathematics Letters, Volume 24, pp. 396-401.

[7] Wang, Z. G., Chen, D. Z. 2009. On a certain subclass of close-to-conex functions: Hacettepe Journal of Mathematics and Statistics, Volume 38, pp. 95-101.

[8] Prajapat, J. K. 2012. Inclusion properties for certain class of analytic functions involving multiplier transformation operator: Journal of Classical Analysis, Volume 1, pp. 35-42.

[9] Prajapat, J. K. 2016. A new subclass of close-to-convex functions: Surveys in Mathematics and Applications, Volume 11, pp. 11-19.

[10] Mustafa, N. 2016. Close-toconvexity of normalized Wright functions: Dokuz Eylul UniversityFaculty of Engineering Journal of Science and Engineering, Volume 18, pp. 290-303.

[11] Panigrahi, T., Murugusundaramoorthy, G. 2016. On Successive Coefficient Estimate for Certain Subclass of Analytic Functions: Applied Mathematics ENotes, Volume 16, pp. 117-124.

[12] Duren, P. L. 1983. Univalent Functions. Grundlehren der Mathematischen Wissenshaften, Bd. 259, New York, SpringerVerlag, 382p.

[13] Goodman, A. W. 1983. Univalent Functions. Volume I, Washington, Polygonal, 246p.

[14] Srivastava, H. M. and Owa, S. (Ed.) 1992. Current Topic in Analytic Function Theory. World Scientific Publishing Company, New Jersey, London, Hong Kong, 456p.
[15] Altıntaş, O. and Owa, S. 1988. On subclasses of univalent functions with negative coefficients: Pusan Kyongnam Mathematical Journal, Volume 4, pp. 41-56.

[16] Moustafa, A. O. 2009. A study on starlike and convex properties for hypergeometric functions: Journal of Inequalities in Pure and Applied Mathematics, Volume 10, pp. 1-16.

[17] Porwal, S. and Dixit K. K. 2013. An application of generalized Bessel functions on certain analytic functions: Acta Universitatis Matthiae Belii series Mathematics, pp. 51-57.

[18] Siverman, H. 1975. Univalent Functions with Negative Coefficients: American Mathematical Society, Volume 51, pp. 106-116.

[19] Altıntaş, O. 1991. On a subclass of certain starlike functions with negative coefficient: Mathematica Japonica, Volume 36, pp. 489-495.

[20] Altıntaş, O., Irmak, H. and Srivastava, H. M. 1995. Fractional calculus and certain starlike functions with negative coefficients: Computers and Mathematics with Applications, Volume 30, pp. 9-16.

[21] Altıntaş, O., Özkan, Ö. and Srivistava, H. M. 2004. Neighbourhoods of a Certain Family of Multivalent Functions with Negative Coefficients: Computers and Mathematics with Applications, Volume 47, pp. 1667 1672.

[22] Porwal, S. 2014. An application of a Poisson distribution series on certain analytic functions: Journal of Complex Analysis, Article ID 984135, pp. 1-3. 